\section{Electron microscopic observation in case of platelet activation in a chronic haemodialysis subject}

\author{
Marianne Schoorl, ${ }^{1}$ Piet C.M. Bartels, ${ }^{1}$ \\ Mareille Gritters, ${ }^{2}$ Donna Fluitsma, ${ }^{3}$ \\ René Musters, ${ }^{4}$ Menso J. Nubé ${ }^{2}$ \\ 1Department of Clinical Chemistry, \\ Haematology and Immunology, Medical \\ Center Alkmaar, Alkmaar; ${ }^{2}$ Department \\ of Nephrology; ${ }^{3}$ Molecular Cellbiology \\ and Immunology; ${ }^{4}$ Physiology, VU \\ Medical Centre, Amsterdam, the \\ Netherlands
}

\section{Abstract}

During haemodialysis (HD), platelets (PLTs) are activated and release granule contents. As HD treatment occurs three times a week, it has been demonstrated that PLTs are exhausted due to the repetitive character of the treatment. To identify PLT depletion morphologically, PLT evaluation was performed by light microscopy and electron microscopy (EM) in a chronic HD subject and a healthy reference subject. Blood samples were taken before the start of HD treatment for measurement of PLT count, PLT volume and size parameters. Blood smears were screened by light microscopy for qualitative evaluation of PLT granule containing cytoplasm, as indicated by its staining density. Morphological PLT parameters of surface area and size of dense bodies were assessed by EM. Data were compared with results of a group of 20 chronic HD subjects and a group of 20 healthy reference subjects. With respect to the percentage of PLTs with appropriate staining density ( $>75 \%$ ), light microscopic evaluation showed that this value (9\%) was within the range of a group of chronic HD subjects, but considerably below the reference range (70\%). EM evaluation revealed an average PLT surface area and dense bodies area of respectively $42 \%$ and $31 \%$, if the healthy reference subject was set on $100 \%$. PLTs from a chronic HD subject are considerably smaller and substantially less granular than PLTs from a healthy reference subject. These findings support the hypothesis of PLT depletion in chronic HD subjects due to frequent PLT activation and/or increased urea concentrations.

\section{Introduction}

As a consequence of extracorporeal blood circulation activation of PLTs occurs in subjects treated with haemodialysis (HD). PLTs are activated due to contact with artificial membranes during HD treatment. The process of PLT activation results in exposure of CD62p, an alpha and dense granule labelling membrane protein, on the outside of the PLT membrane and release of PLT granule content. Concentrations of Platelet Factor 4 (PF4), bthromboglobulin (b-TG) and serotonin in plasma are indicative for the degree of activation of PLTs. ${ }^{1-3}$

As HD-induced PLT activation and degranulation occur three times a week, it has been suggested that PLTs from chronic HD subjects are continuously exhausted due to the repetitive character of the treatment. Recently we demonstrated in a light microscopic study that the staining density of the granule containing cytoplasm of PLTs was considerably reduced., ${ }^{4,5}$ Whereas $70 \%$ of PLTs revealed appropriate granule staining density in healthy subjects, in HD subjects this proportion amounted only to $19 \%$ on average. To observe the morphological aspects of PLT depletion in more detail, both an EM and a light microscopic evaluation of PLTs is performed. Results are compared with a healthy reference subject. In addition, blood samples are taken for haemocytometric analysis of PLTs and compared with recent results in groups of chronic HD subjects and healthy reference subjects. ${ }^{4,5}$

\section{Materials and Methods}

\section{HD subject and healthy reference subject}

Particularly for EM, one representative HD subject of a group of 20 chronic HD subjects was selected. ${ }^{4,5}$ The patient (woman, age 57 ) underwent 44 months for three times a week HD treatment with a low flux polysulphone ${ }^{\circledR} \mathrm{F} 8$ membrane (Fresenius, Bad Homburg, Germany). As anticoagulant Fragmin ${ }^{\circledR}$ (intravenously $3500 \mathrm{U}$ bolus injection) was used. The original kidney disease was due to hypertension. She did not use coumarines, acetylsalicylic acid, clopidrogel, non-steroidal antiinflammatory drugs or related compounds. Blood samples were collected from the arterial line before starting $\mathrm{HD}(\mathrm{t}=0)$.

The representative healthy subject of a group of 20 healthy reference subjects, was a healthy 45 years old female laboratory technician without a medical history and without any medication. ${ }^{4,5}$

\section{PLT counts, PDW, MPV, and p-LCR}

Blood samples were collected in $\mathrm{K}_{2}$ EDTA tubes (Vacutainer, Becton Dickinson, Plymouth, UK). PLT count, PLT distribution width (PDW), mean PLT volume (MPV) and
Correspondence: Marianne Schoorl,

Department of Clinical Chemistry, Haematology and Immunology, Medical Center Alkmaar, Wilhelminalaan 12, 1815 JD Alkmaar, The Netherlands.

Tel. +31.72.5483632 - Fax +31.72 .5482175$

E-mail:m.i.schoorl@mca.nl

Key words: electron microscopy, platelet activation, platelet degranulation, haemodialysis.

Contributions: MS design, analysis of hemocytometric parameters, microscopic platelet evaluation, data interpretation; PCMB design, data interpretation, intellectual content of PLT activation and PLT morphology; MG design and content of PLT activation and hemodialysis; DF microscopy analysis; RM microscopy analysis and intellectual content of electron microscopy; MJN data interpretation, intellectual content of PLT activation, hemodialyis and importance of the work.

Conflict of interest: the authors report no conflicts of interest.

Received for publication: 29 July 2011.

Accepted for publication: 16 September 2011.

This work is licensed under a Creative Commons Attribution NonCommercial 3.0 License (CC BYNC 3.0).

(C) Copyright M. Schoorl et al., 2011

Licensee PAGEPress, Italy

Hematology Reports 2011; 3:e15

doi:10.4081/hr.2011.e15

PLT large cell ratio (p-LCR) were measured using a Sysmex XE-2100 haematology analyser with a dedicated IPF-Master software package (Sysmex Corporation, Kobe, Japan).

\section{PLT morphology}

Two peripheral blood smears were prepared for evaluation of PLT morphology and stained according to May-Grünwald-Giemsa methodology on a Sysmex SP-100 analyser (Sysmex Corporation, Kobe, Japan). The slides were light microscopically screened for qualitative evaluation of morphological aspects of PLTs with application of a Cella Vision ${ }^{\mathrm{TM}}$ DM96 analyser (Cella Vision AB, Lund Sweden). Using the Cella Vision ${ }^{\mathrm{TM}}$ DM96 it is possible to reduce deviations between observations of biomedical scientists. Granule-containing cytoplasm, indicated as a granulomere, is stained light purple or pink. After discharge of granule content, activated PLTs are faintly stained grey. ${ }^{6}$ Qualitative aspects were evaluated by classification of the PLT content in four categories corresponding to a staining density of granule-containing cytoplasm amounting to $<25 \%, 25-50 \%, 50-75 \%$ or $>75 \%$, respectively. Staining density of $>75 \%$ of the granule-con- 
taining cytoplasm in $>50 \%$ of PLTs was considered to be appropriate (Figure 1).

\section{Electron microscopy}

PLTs were fixed in $2 \%$ glutaraldehyde in $0.1 \mathrm{M}$ sodiumcacodylate buffer PH 7.4. After postfixation in $1 \%$ osmiumtetroxide in sodiumcacodylate buffer, PLTs were pelleted in 1\% agar and subsequently dehydrated in ethanol and embedded in Agar 100 Resin (Agar Scientific, Stansted, UK). Ultrathin sections were stained with uranylacetate and leadcitrate and examined with application of a transmission electron microscope (TEM), model Philips CM100 Bio Twin (Philips/FEI Corporation, Eindhoven, The Netherlands).

\section{Image analysis}

Electron micrographs were imported as Tag Image File Format (TIFF) and analyzed with application of dedicated Slidebook ${ }^{\mathrm{TM}}$ image analysis software (Slidebook version 4.2; Intelligent Imaging Innovations (3I), Denver, C0, USA). Platelet morphology parameters (e.g. cross sectional surface area, xy-shape and number and size of dense bodies per platelet) were derived from so called digital image masks, binary overlays which were created in an operator-independent fashion based on fixed threshold settings. Subsequently, individual mask objects were analyzed and quantified. In each subject, 20 regions of interest were analyzed yielding $>250$ PLTs per individual.

\section{Results}

\section{Haemocytometry}

As can be seen from Table I, the haemocytometric data of the selected HD subject, reflecting PLT volume and size characteristics, are within the range of the group of 20 chronic HD subjects and almost comparable to the selected healthy reference subject / reference ranges.

\section{Light microscopic evaluation}

Light microscopic evaluation of PLTs with appropriate $(>75 \%)$ and with decreased $(<25 \%)$ staining density of the granule-containing cytoplasma is shown in Figure 1. In the subject with chronic HD treatment appropriate staining density of the granule containing cytoplasm is decreased to a minimum of $8 \%$ if compared with the reference ranges for healthy controls (mean 70\%, range 44-86\%), but within the outer limits of the group of $20 \mathrm{HD}$ subjects (mean 19\%, range 4-41\%) (Table 1).

\section{Electron microscopic evaluation}

EM photographs of PLTs of the HD subject and healthy reference subject are shown in Figure 2. Evaluation revealed an average PLT surface area only of 242.481 pixels in the HD subject (Figure 2/A) and 576.533 pixels in the healthy reference subject (Figure 2/B). The proportional area of dense bodies per PLT, set at $100 \%$ in the healthy reference subject, was $31 \%$ in the HD patient (Table 1).

\section{Discussion}

In the present study, several PLT characteristics are assessed in a representative HD subject of a group of 20 chronic HD subjects, who did not use any drugs interfering with PLT activation. It is demonstrated, that the PLT surface area, as assessed by EM, was considerably smaller in case of the HD subject (42\%) than in the healthy reference subject. In comparison with the healthy reference subject, the area of dense bodies per PLT amounted to $31 \%$. The staining density of PLTs, reflecting the amount of granule-containing cytoplasm as

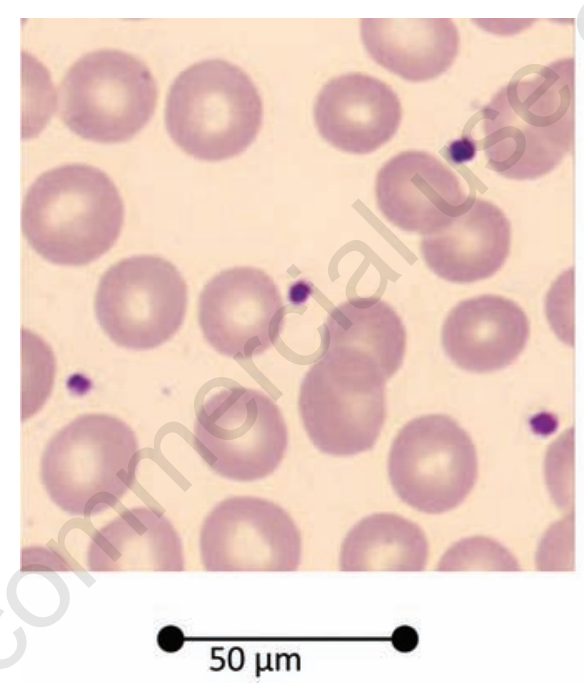

assessed by application of light microscopy in the HD subject, is obviously below the result of the reference subject. These data suggest either a failure in the synthesis and storage of granule contents, and/or preceding PLT degranulation. Indeed, as previously demonstrated by our group and others, PLT granule products like PF4, $\beta$-TG and serotonin are released during HD. ${ }^{5,7}$ As these phenomena occur three times a week, month after months, it has been suggested that PLTs from chronic HD subjects are depleted and, in combination with the uremic environment, persistently deficient. ${ }^{8,9}$

Size and volume parameters MPV, PDW and p-LCR of the HD subject are within the range of a group of chronic HD subjects. From the current study it is not immediately apparent why the decline in PLT volume, as derived from digital image masks of EM evaluation, markedly exceed the small alterations in PLT volume as assessed by haemocytometry. It may result

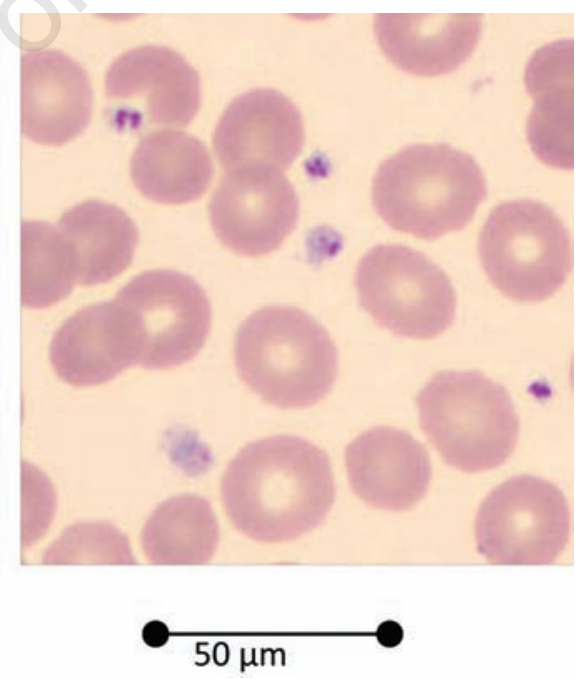

Figure 1. Light microscopic evaluation of PLTs with appropriate $(>75 \%$, left) and with decreased ( $<25 \%$, right) staining density of the granule-containing cytoplasma. Magnification: 600x.
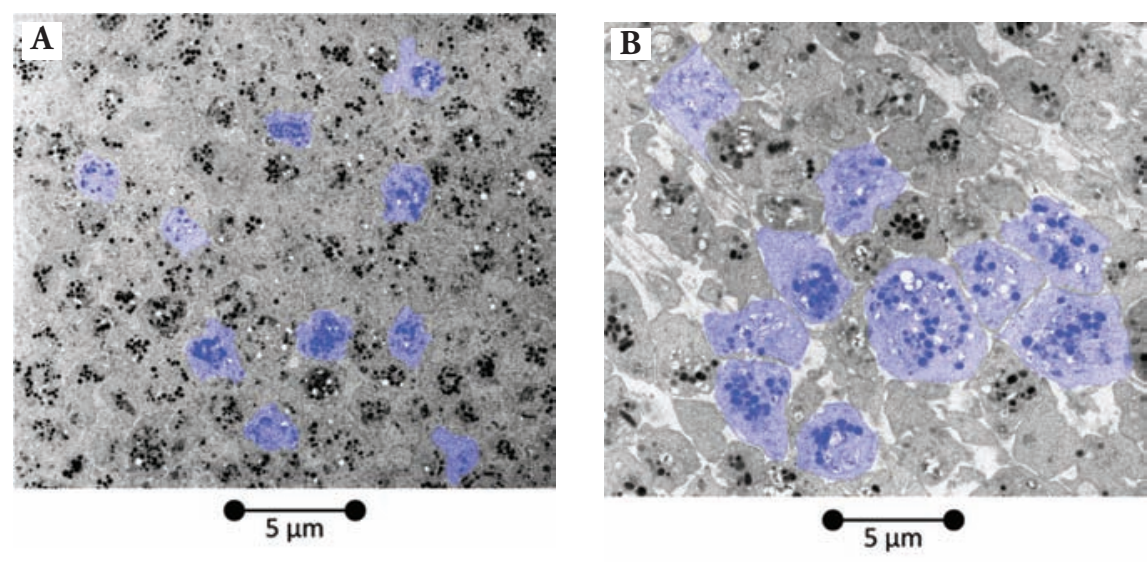

Figure 2. (A) Digital mask of electron microscopic PLT evaluation of a HD subject and (B) healthy reference subject. Masks are shown in blue. Magnification: 2500x. 
Table 1. Laboratory, light microscopic and EM data from a HD subject and healthy reference subject. For comparison data of the groups of 20 chronic HD subjects and 20 healthy reference subjects are used. ${ }^{4}$

\begin{tabular}{|c|c|c|c|c|}
\hline & $\begin{array}{l}\text { HD } \\
\text { subject }\end{array}$ & $\begin{array}{l}\text { HD subjects' } \\
\text { group }^{3} \\
\text { (mean, range) } \\
\mathrm{N}=20\end{array}$ & $\begin{array}{l}\text { Healthy } \\
\text { reference } \\
\text { subject }\end{array}$ & $\begin{array}{l}\text { Healthy reference } \\
\text { group }{ }^{3} \\
\text { (mean, range) } \\
\mathrm{N}=20\end{array}$ \\
\hline $\begin{array}{ll}\text { PLT } & \left(10^{9} / \mathrm{L}\right) \\
\text { PDW } & (\mathrm{fL}) \\
\text { MPV } & (\mathrm{fL}) \\
\text { P-LCR } & (\%)\end{array}$ & $\begin{array}{l}206 \\
11.3 \\
10.0 \\
23.4\end{array}$ & $\begin{array}{l}198(117-229) \\
9.5(7.7-11.4) \\
10.5(9.1-11.8) \\
29.0(16.9-40.1)\end{array}$ & $\begin{array}{l}254 \\
12.1 \\
10.3 \\
27.2\end{array}$ & $\begin{array}{l}238(150-337) \\
11.7(9.5-14.1) \\
10.1(8.9-11.6) \\
25.2(15.2-37.3)\end{array}$ \\
\hline $\begin{array}{l}\text { Light microscopic evaluation: } \\
\text { \% PLTs with > 75\% } \\
\text { staining density }\end{array}$ & 8 & $19(4-41)$ & 72 & $70(44-86)$ \\
\hline $\begin{array}{l}\text { EM evaluation: } \\
\text { average PLT surface } \\
\text { area (\# pixels) } \\
\text { area of dense bodies } \\
\text { per PLT (\%) }\end{array}$ & 242.481 & & 576.533 & \\
\hline
\end{tabular}

from decreased activity of megakarypoiesis accompanied with PLT volume reduction as a result of frequent PLT activation. Also, increased urea concentrations may reveal an additional cause for occurrence of PLTs with reduced density of granules in the cytoplasm. In this respect it is interesting to note that the masking procedure in this study was relatively difficult to perform due to the clustering of PLTs in several electron micrographic TIFFs. Possibly, PLTs in an uraemic environment may be extremely sensitive for the fixation procedure applied for EM, resulting in abnormal dehydration and shrinkage.

In conclusion, EM evaluation showed that PLTs from an HD subject are considerably smaller and substantial less granular than
PLTs from a healthy reference subject. These observations support the results of increased amounts of PLTs with decreased staining density of granule-containing cytoplasm by light microscopic evaluation and the hypothesis of PLT depletion in subjects with chronic intermittent HD.

\section{References}

1. Sebekova K, Spustova V, Opatrny K Jr., Dzurik R. Serotonin and 5-hydroxyindoleacetic acid. Bratisl Lek Listy 2001;102:351-6.

2. Bartels PCM, Schoorl M, Schoorl M. Activatie van stolling tijdens hemodialyse is afhankelijk van de wijze van antistolling. Ned Tijdschr Klin Chem Labgeneesk 2005;30:282-4.

3. Schoorl M, Schoorl M, Bartels PCM. Platelet activation and serotonin release during treatment with haemodialysis. Ned Tijdschr Klin Chem Labgeneesk 2006;31: 236-8.

4. Schoorl M, Schoorl M, Bartels PCM. Changes in platelet volume, morphology, and RNA content in subjects treated with haemodialysis. Scand J Clin Lab Invest 2008; $68: 335-42$

5. Schoorl M, Schoorl M, Nubé MJ, Bartels PCM. Platelet depletion, platelet activation and coagulation during treatment with hemodialysis. Scand J Clin Lab Invest 2011;71:240-7.

6. Bain BJ. Blood sampling and film preparation morphology. In Blood Cells: A practical Guide. 2nd ed. Blackwell Science, Oxford, UK. 1995; pp 98-100.

7. Cianciolo G, Stefoni S, Donati G, et al. Intra- and post-dialytic platelet activation and PDGF-AB release: cellulose diacetate vs. polysulfone membranes. Nephrol Dial Transplant 2001;6:1222-9.

8. Thijs A, Nayanakkara PW, ter Wee PM, et al. Mild-to moderate renal impairment is associated with platelet activation: a cross-sectional study. Clin Nephrol 2008;70:325-31.

9. Diaz-Ricart M, Estebanell E, Cases A, et al. Abnormal platelet cytoskeletal assembly in hemodialyzed patients results in deficient tyrosine phosphorylation signaling. Kidney Int 2000;57:1905-14. 